\title{
Functional Nanomaterials: Energy and Sensing
}

\author{
TERRY T. XU ${ }^{1,3}$ and JUNG-KUN LEE ${ }^{2,4}$ \\ 1.-Department of Mechanical Engineering and Engineering Science, The University of North \\ Carolina at Charlotte, Charlotte, NC 28223, USA. 2.-Department of Mechanical Engineering and \\ Materials Science, University of Pittsburgh, Pittsburgh, PA 15261, USA. 3.-e-mail: ttxu@ \\ uncc.edu. 4.—e-mail: jul37@pitt.edu
}

Sponsored by the Nanomaterials Committee, this special topic has a unique combination of short review papers and research papers on some recent advances for using functional nanomaterials for energy conversion/storage and optical/electric/magnetic signal sensing.

The invited paper from Zhu's group is an extension of their talk "One-Dimensional Nanostructures for Wearable Devices" presented at the TMS 2015 Annual Meeting \& Exhibition (TMS2015). The paper reviews nanomaterial-enabled dry electrodes for electrophysiological sensing, particularly on electrocardiography (ECG). It starts with an overview of two different commercial electrodes (i.e., pre-gelled wet electrode and dry electrode), their electrode-skin interface models and the source of noise. The overview shows that dry electrodes have certain advantages over the wet ones, such as the elimination of signal degradation caused by dehydration of the gel. The overview also points out that (1) flexible dry electrodes are needed to form intimate contact with skin surface so that the movement of electrodes and motion artifacts can be suppressed and reduced, and (2) the nanomaterials-based electrode could be the solution. The paper then presents a comprehensive review on recent developments of dry ECG electrodes made of various nanomaterials, including metallic nanowires, metallic nanoparticles, carbon nanotubes and graphene. Their fabrication processes and performances are discussed. The paper concludes that nanomaterials-enabled dry ECG electrodes are attractive candidates for long-term ECG sensing, and calls for more studies in this field.

The invited paper from Zhao's team is a recap of their talk "Detecting Bacteria by Surface Enhanced Raman Spectroscopy" at TMS2015. This paper

Terry T. Xu and Jung-Kun Lee are the JOM advisors for the Nanomaterials Committee of the TMS Functional Materials Division, and guest editors for the topic Functional Nanomaterials: Energy and Sensing in this issue. reports how surface-enhanced Raman spectroscopy (SERS) contributes to food safety by detecting the low levels of pathogenic microorganisms that may exist in foods. In particular, vegetables and fruits are vulnerable to contamination by pathogenic microorganisms since they are natural vehicles of pathogens from cultivation to delivery. Therefore, to detect a small amount of harmful microorganisms is a very important, but challenging task for food safety. Compared with traditional Raman spectroscopy, SERS exploits novel metal nanostructures to enhance the sensitivity. In this study, the authors demonstrate that the limit of detection (LOD) of Salmonella Poona from cantaloupe cubes can be as low as $100 \mathrm{CFU} / \mathrm{ml}$ in less than $4 \mathrm{~h}$ by performing SERS measurements on vancomycin-coated silver nanorod (VAN AgNR) substrates. It is claimed that such a small LOD has not previously been reported for label-free, intrinsic SERS detection using real food samples. This result suggests that the SERS detection technique based on AgNR is a powerful platform for a rapid, simple, and inexpensive means of pathogenic bacteria detection, which will be very useful in securing the safety of fresh foods.

Bimetallic nanoparticles, composed of two different metal elements, show distinctive properties as compared to those of monometallic nanoparticles. They have received considerable attention because of their potential applications in magnetic, optical, and catalytic fields. Xiong et al. report their recent work on the synthesis and optical characterization of silver-copper $(\mathrm{Ag}-\mathrm{Cu})$ bimetallic nanoparticles. The nanoparticles were synthesized by the reduction of silver nitrate and copper (II) acetate monohydrate in ethylene glycol. Both microwave-assisted heating and conventional oil bath heating methods were employed. The results showed that the formation of Ag-Cu core-shell bimetallic nanoparticles, and in turn the optical properties, were strongly affected by the heating method. More specifically, the microwave-assisted method could increase the rate of $\mathrm{Cu}$ reduction, lead to the formation of a less 
homogenous $\mathrm{Cu}$ shell on the $\mathrm{Ag}$ core, and produce a broad extinction spectrum with two distinct peaks at wavelengths of around $400 \mathrm{~nm}$ and $600 \mathrm{~nm}$.

New treatment for bacteria is becoming more important, because traditional methods have led to the emergence of bacterial strains which are not easily killed. Some bacteria such as Staphylococcus aureus have shown their ability to develop resistance to several common antibiotics. Gene mutation resulting from the exposure of bacteria to antibiotics costs a significant number of human lives every year. For example, in 2009, 440,000 new multidrugresistant tuberculosis cases $(3.3 \%$ of all new tuberculosis cases) were reported and 150,000 patients died because of multi-drug resistant Mycobacterium tuberculosis. ${ }^{1,2}$ One solution to antibacterial resistance is to use the photocatalytic activity of nanostructured semiconductors such as $\mathrm{TiO}_{2}$. When $\mathrm{TiO}_{2}$ nanoparticles are exposed to ultraviolet (UV) light, photon energy is absorbed and electron/hole pairs are generated in the conduction and valence bands. These photogenerated carriers can oxidize microorganisms both directly or indirectly (via mediator hydroxyl radical). The paper by O. Obidi and L. Halverson reports the effect of an illumination source on the antimicrobial properties of $\mathrm{TiO}_{2}$ nanoparticles. They picked Bacillus sphaericus and Klebsiella pneumoniae as models for Grampositive and Gram-negative microorganisms that are routinely isolated from spoilt paints. The results in this paper demonstrated the loss of viability of the test organisms in the presence of $\mathrm{TiO}_{2}$ nanoparticles using a culturable (plate) count technique. A obvious decrease in the numbers of viable $B$. sphaericus and $K$. pneumoniae was observed after the bacteria were exposed to light for $24 \mathrm{~h}$. In addition, fluorescent light containing a more visible light component causes more effective anti-microbial activity of the $\mathrm{TiO}_{2}$ nanoparticles, which implies the synergetic effect of UV and visible lights in dealing with resistant bacteria. This paper shows a potential antibacterial application of the photocatalysis of $\mathrm{TiO}_{2}$ in the paint industry, since $B$. sphaericus and $K$. pneumoniae are readily found in spoilt paints.

The following papers being published under the topic of Functional Nanomaterials: Energy and Sensing provide excellent details and research on the subject. To download any of the papers, follow the url http://link.springer.com/journal/11837/68/4/ page/ 1 to the table of contents page for the April 2016 issue (vol. 68, no. 4).

- "Nanomaterial-Enabled Dry Electrodes for Electrophysiological Sensing: A Review" by Shanshan Yao and Yong Zhu.

- "Rapid Detection of Pathogenic Bacteria from Fresh Produce by Filtration and Surface Enhanced Raman Spectroscopy" by Xiaomeng Wu, Caiqin Han, Jing Chen, Yao-wen Huang, and Yiping Zhao.

- "Effect of Synthesis Techniques on Crystallization and Optical Properties of Ag-Cu Bimetallic Nanoparticles" by Ziye Xiong, Fen Qin, Po-Shun Huang, Ian Nettleship, and Jung-Kun Lee.

- "Photoinactivation and Toxicity of Nano-sized $\mathrm{TiO}_{2}$ on Paint Microflora Using Visible Lights" by Olayide Obidi and Larry Halverson.

\section{REFERENCES}

1. Antimicrobial Resistance, Global Report on Surveillance (Geneva: WHO Press, 2014).

2. M. Adhvaryu and B. Vakharia, Clin. Pharmacol. 3, 51 (2011). 